noticed that in these five pairs the temperature of the mouth of both of us was almost the same, but that four times out of the five my surface temperature was higher than his-on two occasions (the second and third) as much as $3.6^{\circ} \mathrm{F}$. A possible explanation for the fact that on the fourth occasion my surface temperature was lower than his is that we made the observations in his house, which is about 200 yards from mine, and on the evening on which this observation was made I strolled to his house after dinner while there was a cold north-east wind blowing. On four out of the five occasions I sweated more than he did. The whole amount I perspired during the five observations was 15.3 milligrammes, but his

Table XIV. C.-Yotng Man with Ague.

\begin{tabular}{l|c|c}
\hline Time. & Volume of arm. & Temp. (Deg. C.). \\
\hline 11.0 & 60 & $39 \cdot 9$ \\
11.10 & 62 & $40 \cdot 3$ \\
11.30 & 60 & $4 c \cdot 6$ \\
11.45 & 63 & $40 \cdot 6$ \\
12.0 & 76 & $40 \cdot 6$ \\
12.15 & 74 & $40 \cdot 6$ \\
12.30 & 78 & $40 \cdot 5$ \\
12.45 & 82 & $40 \cdot 3$ \\
1.0 & 32 & $40 \cdot 4$ \\
1.15 & 90 & $40 \cdot 2$ \\
1.30 & 96 & $40 \cdot 0$ \\
2.0 & 108 & $40 \cdot 0$ \\
2.30 & 120 & $40 \cdot 0$ \\
3.0 & 126 & $39 \cdot 8$ \\
3.30 & 133 & $39 \cdot 7$ \\
4.0 & 138 & $39 \cdot 5$ \\
4.30 & 143 & $39 \cdot 4$ \\
5.0 & 149 & $38 \cdot 3$ \\
\hline
\end{tabular}

total amount was only $11 \cdot 1$, so that I perspired 38 per cent. more than he did. As, therefore, my surface temperature was higher and the amount of sweat that I secreted was greater I lost heat from radiation, conduction, and evaporation faster than he, and therefore my production per kilogramme was greater than his. We consequently learn that even healthy individuals vary much in their production and loss of heat. The last pair of observations is of little value, as the temperature of our baths was not the same. I have given in the table the temperature of the air, taken with both a wet and dry bulb thermometer. The air was driest on April 11th, on which day I sweated most, but this was not the day that Dr. Garrod sweated most; and on the next driest day I sweated less than on any other, so as far as these few observations go they do not show any relation between the humidity of the air and the amount of sweat secreted.

It is well known that muscular contraction is accompanied by a rise of temperature; therefore, were it not that the loss of heat is increased during exercise the temperature would always rise. In fact, often the increased loss does not compensate for the increased production, and so the temperature does rise. Many references to this subject are given by Pembrey. ${ }^{5}$ Jurgensen found that the work involved in sawing wood for six hours raises the temperature of a healthy $\operatorname{man} 1.2^{\circ} \mathrm{C}$. above normal. Davy showed that walking for two or three bours raised the temperature of the urine $0.8^{\circ} \mathrm{C}$. Clifford Allbutt found that Alpine climbing raised the temperature of the mouth half a degree, and Liebermeister and Hoffman found their axillary temperature raised by the exertion both of coming up and going down a mountain. Hobday, too, has taken the rectal temperature of omnibus horses, and he finds that it is raised about $2^{\circ} \mathrm{C}$. by hard work. Dr. Garrod and I thought, therefore, it would be of interest to make a series of observations on the effect of exercise on each other (see Tables XVI. and XVII.). It will be seen that a bicycle ride of seventeen miles in a high, dry, cold wind reduced the internal temperature of $\mathrm{Dr}$. Garrod by $1 \cdot 0$, and my own by 0.8 of a degree I do not think this can have been due to the inhalation of cold air cooling the mouth, for we did not take the temperature of the mouth until we had been in the house for twenty

$$
5 \text { op. oit. }
$$

minutes after the ride, and it must have been due to the rapid loss of heat caused by our going quickly through the high, dry, cold wind. With the exception of this bicycle ride it will be seen that exercise always raised Dr. Garrod's internal temperature and that mine was either unaltered or raised, but that mine was, on the whole, much less affected than his. There are other interesting points of contrast between us. Dr. Garrod's surface temperature was always considerably raised by exercise, while mine was usually slightly diminished. On the other hand, he sweated but little in comparison with me, for his arerage increased amount of sweat was 3.5 millligrammes, while mine was 7.05 . The difference is really greater than this, for I took two bicycle rides in a cold, dry wind to his one, and in a very dry wind the sweat evaporates fast, and consequently not much is secreted when the observation is taken. Apparently, therefore, we are different in our methods of accommodating the loss of heat to the increased production dne to the muscular exercise; he chiefly increased bis radiating and conducting power; I chiefly increased my loss by evaporation. We have therefore a demonstrated proof that different people accommodate differently. These tables, too, show strikingly what Table XV. has already shownnamely, that when at rest my surface temperature is always higher than Dr. Garrod's, and that I perspire more than he does; in other words, I lose heat faster and therefore must produce more calories per kilogramme. As in fever, so here the data are not sufficient for us to say how many calories of heat the muscular exercise causes to be produced-we can only say that it must at least have been more than a certain number, for instance, my weight without $m y$ clothes is 61 kilogrammes; supposing the effect of exercise was to raise my temperature $0.5^{\circ} \mathrm{C}$., and the loss by radiation, conduction, and evaporation were all increased, then $I$, as soon as when taking exercise I had raised my temperature the half a degree Centigrade, must have produced at least more than an additional 30.5 kilogramme Centigrade calories, as my specific heat is nearly that of water.

\section{ON SOME SUPPURATIONS OF THE URINARY APPARATUS. ${ }^{1}$}

BF REGINALD HARRISON, F.R.C.S. ENG., SURGEON TO ST. PETER'S HOSPITAL; PRESIDKNT OF THE MEDICAL SOCIETY OF LONDON.

I HAVE selected for consideration some points which seem to arise out of the pathology of certain suppurative processes as observed in the urinary organs in relation to the more modern treatment of surgical lesions and infections as generally practised. I shall first illustrate my observations by taking the most common, as well as the farthest reaching, cause of suppurations of the urinary organs, as occurring in the form of primary gonorrhceal infections in the male and the female, and as secondary developments in other parts of the genito-urinary system, as more frequently observed in the latter sex. The advanoes that have been made in connexion with the bacteriology of the urinary organs in reference to the kind and extent of these infections have already led to some useful applications in the way of treatment which I will briefly notice. The investigations of Guyon, Janet, Hallé, and others of the French school, more particularly as regards the life-history and cultivation of these microorganisms, are of much interest and importance. It is, however, to the practical aspect of the subject that I shall confine my remarks. The first point that strikes one is that many of the cases spoken of as instances of chronic suppurative uretbritis or gleet represent a far more extensive invasion than is generally supposed to be the case. In this lies, I have no doubt, an explanation of the difficulty that is not infrequently experienced in bringing cases of this kind to a successful and speedy termination. Many gleets are merely re-infections of the urethra from a bladder which serves the purpose of a medium for bacterial cultivation. So long as a gonorrhoea is confined to the area of the urethra as defined by that portion of the canal which lies in front of the compressor urethræ or "cut off" muscle, 1 A papar read bef re the Medical Sosiety cf Erixt on on June 10th,
1897. 
the task of successfully combating the infecting suppuration is not usually a difficult one. The disease is withın limits which can be readily acted upon by various agencies, administered either locally or by internal medication, and its course is generally limited both in severity and duration. When, however, these boundaries are exceeded and the bladder becomes invaded it is not easy to prevent re-infection taking place as if from an extraneous source. Hence the disease may be almost indefinitely protracted. Nor is invasion of the bladder in this way uncommon or necessarily indioated by acute symptoms such as would unmistakeably draw atten. tion to the occurrence. In most instances of this the prostate is the only part of the urinary apparatus that may show any active indication of contact with the septic products, the mucous membrane of the bladder, though harbouring them, being singularly insensitive to bacterial influences. That infection occurs in this way can be readily demonstrated. When the b!adder has been thus involved the conditions of the urinary organs, particularly during the night, are extremely favourable for bacterial cultivations, a circumstance which may explain the constancy and degree of matutinal suppuration exaded by the urethra which is a prominent symptom in this class of cases. It is rare, I believe, for gonorrhoeal organisms to make their way up the ureters and to invade the kidneys-a fact which is no doubt due to the guarded manner in which these ducts enter the bladder and the constancy of the downward flow of the urine. That renal infection and urethral inflammation sufficient to cause a pyelitis of the former and a contraction or stricture of the latter can occur has been demonstrated as p jssible. Some years ago I examined the body of a middleaged man who died from acate single pyelitis rapidly follow. ing upon a first attack of gonorrbœx. The ureter of the kidney involved was extremely dilated, a circumstance which was believed to be due to the patient having been in the habit for some years previously of passing renal calculi The opposite kidney was much congested. Mr. W. G. Nash ${ }^{2}$ has recorded a case of strictured ureter which was probably caused in this way. In the female the widespread effects of the gonorrhoeal infection are generally recognised.

The suspicion, however, that the bladder may be infected with micro-organisms should be made a matter of proof, and this can generally be done. For this purpose care should be taken to obtain a specimen of the urine as it exists in the bladder, minus any contaminations it may pick up in its transit along the urethra. The first portion of the urine passed should be rejected as probably containing the flushings of the canal, the balance being separately collected as representing the constituents of what may be called resical urine. When it is possible a catheter specimen should be obtained, in accordance with the directions of the Clinical Research Association, where there are reasons for believing that the bladder is infected. In this way gonococci may be detected in resical urine in cases of gonorrhœa. The mere fact that the urine is shown to contain bacteria does not necessarily imply that the individual is capable of inoculating another with gonorrhœx. Where, however, the gonococcus is discovered in the urine or the discharges of the patient, as is not unfrequently the case, I d o not think there can be any doubt as to the possibility of its communication to some one else. The bacteriological examination of these secretions may furnish important evidence in determining whether a person who has been comparatively recently infected should be allowed to marry. So long as these conveyers of infection can be detected in the urine or the discharges, so long may we expect that transference of the disease is liable to follow. In these cases of secondary infection of the bladder, caused by extension from the urethra, I have for some time placed much reliance upon the irrigation of the bladder and urethra with antiseptics, as described by Dr. Janet, ${ }^{3}$ with some modifivations. The object is attained in the following manner. The apparatus consists of an ordinary hydrostatic bladder tank, holding about one pint of water and fitted with a nozzle to which a No. 8 Jacques' rubber catheter can be attached. The tank is elevated about six feet from the ground, and is filled with warm water containing 30 minims of Condy's fluid to a pint of the latter. For lubricating the catheter I use carbolised vaseline. The patient having emptied his bladder spontaneously is placed in the recumbent position and the catheter is then passed. Before the nozzle of the irrigator is connected with the catheter $\mathrm{s} s$ it lies in the bladder the fluid is allowed to run off for a few seconds so as to ensure that there is no free air in the tube of the apparatus. Then the connexion is made and the fluid is allowed to flow into the bladder by degrees until the patient is conscious of feeling distention. I generally use from twelve to sixteen ounces for this purpose, allowing it to enter the bladder in jets of about three or four ounces at a time. In this way the feeling of any sudden or extreme fulness is avoided, and the entire area of the mucous membrane of the bladder is unfolded and opened out, and thus comes in contact with the permanganate solution. When a sufficient degree of bladder distension is obtained the catheter is slowly removed, care being taken not to allow the fluid to escape. I then usually lightly palpate the bladder above the pubes with the hand before the patient stands up. This he should then do and proceed to empty his bladder of its contents by his natural efforts. Thus not only is the bladder washed out, but the whole urethra is flushed in a manner that is impossible by any other artificial method. As the patient is voiding the contents of his bladder it is well to direct him to suddenly interrupt the outflow once or twice by pressure with the finger on the penile urethra. In this way the lacunæ of the canal are also distended and flushed by the irrigating fluid. This completes the process, which may be repeated once or twice in the twenty-four hours, until the urine and the urethral mucus are found free from organisms. Most patients after proper instruction will be able to carry out all these details. On the conclusion of each irrigation the patient should rest for a time in the recumbent position. I have only referred to the permanganate of potash in great dilution as the flushing agent for use in these cases. Half a drachm of Condy's solution, as I have already mentioned, gradually increased to a dracbm in a pint of water, has given me good results. In other instances the preparation known as "Sanitas," in the proportion of an ounce to a pint of water, has also answered equally well. Nentral sulphate of quinine (1 grain to the ounce) may also be used. More rarely I have employed nitrate of silver (onesixteenth grain to the ounce) and perchloride of mercury. The last sometimes produces a good deal of pain, though only used in the proportion of 1 in 10,000 . I have no doubt for aborting infections the perchloride is the most effective. When used for the latter purpose in the proportion of 1 in 10,000, and after the solution bas been spontaneously voided from the bladder as in the act of micturition, the bladder may be filled with a strained solution of albumin-say, that yielded by one fresh egg-in a pint of tepid or nearly cold water. If this is done and the albuminoid solution voided in like manner from the bladder, any pain, smarting, or spasm that the perchloride solution produced at once ceases. Similarly, a weak solution of chloride of sodium, injected into the bladder after a solution of nitrate of silver has been used for this purpose, not only removes any irritation the latter may provoke, but coats over the inflamed mucous membrane with a thin layer of chloride of silver, which of ten affords much relief when cystitis is present. Probably there is no better bactericide than nitrate of silver in these cases, but it requires to be used with the precautions mentioned when good results may be obtained. In this class of cases where suppuration proceeds directly from infection the internal administration of bactericides may be advantageously combined with the local treatment just referred to. In rendering the urine sterile and destructive to the life of the gonococcus there are no more effectual agencies than some of those which are derived from the vegetable kingdom. I refer particularly to the volatile oils which are so largely eliminated by the kidneys of which I may instance sandal wood, copaiba, cubebs, and similar drugs. By these it is possible to so saturate the urine as to render the existence of certain forms of bacteria impossible. I shall, however, refer later to other methods of sterilising the urine which may also be utilised in the treatment of infecting forms of urethritis and cystitis.

I will now notice other varieties of urinary suppurations which may be best illustrated by certain cases of prostatic obstruction. Though the urine is in these instances usually puralent and charged with bacteria the latter are not infective in the same sense as those I have previously referred to in connexion with a specific form of urinary suppuration. On the other hand, it must be remembered that these microorganisms are readily capable of transference from one individual to another through the medium of catheters and 
instruments of this kind, and thus they may be regarded as factors in the causation of extensive and far-reaching suppurations in these parts. The principles of treatment in this class of suppurations are the same as those I have already mentioned, subject to certain modifications which I will proceed to notice. Putting aside the consideration of the precise means which these cases often require for mechanically emptying the bladder and thus preventing decomposition of the urine taking place, the restoration of the secretion to its normal state will be mainly brought about by antiseptics in the manner indicated. The more or less atonic condition of the bladder in these obstructive cases seldom permits us to employ the process of autoirrigation as previously described, and we must therefore for this purpose avail ourselves of one or other of the methods usually practised. In offering a few remarks on the toilette of the bladder I will do so in reference-(1) to the disposition of the parts requiring ablution; (2) to the mechanism employed; and (3) to the composition of the cleansing fluid. Most of us, I think, will admit that we are disposed to draw our ideas relative to the process of washing out the bladder from the natural state of the parts such as we see in anatomical plates. If, however, we select for our guidance the majority of pathological specimens illustrating the obstructive diseases of these parts we shall at once recognise the diffeulties that beset us in our endeavour to wash out these, as we should do, for instance, in the case of a pint vessel. Take, for example, the hypertrophied bladders, with their various saccules, pouches, and dependencies, which are met with in connexion with advanced urethral stricture in younger adults and in persons of more advanced age who are the subjects of obstructive prostatio disease. In many of these the bladder is no more like the natural organ than the interior of a glass bowl resembles the section of a coarse bath sponge. The contents of the latter you could hardly ever hope to wash out effectually, though you may succeed in soaking them out. For washing out the bladder I almost invariably employ the hydrostatic tank which is now so generally used. It has, I believe, many advantages over syringes which I need not here stop to enumerate. When the bladder is much fasciculated it should be done in the recumbent position, with the object of opening out the mucous membrane as completely as possible without causing pain, the object being to reach irregularities on the surface which otherwise would escape contact with the lotion. When the bladder is much pouched it is a good plan, after filling it and before removing the fluid, to cause the patient to agitate his body from side to side so as to bring any dependencies there may be within reach of the antiseptic. In various ways such as these the process of washing out the bladder may be rendered more searching. For washing out in ordinary cases of bacterial urine I usually prefer boracic acid or the boroglyceride. Condy's fluid, sanitas, and iodoform may also be similarly employed. As I bave already said, a solution of nitrate of silver in the proportion of $\frac{1}{16}$ of a grain to the ounce is often efficacious in rendering turbid urine clear after other things have failed. The sterilisation of the urine by internal medicines is an important adjunct in the treatment of the class of cases $I$ am now referring to. It is quite unnecessary to draw attention to the many obvious ways in which the urine may thus be altered. The specific gravity, the reaction, and the composition of this secretion may to a large extent be artificially influenced, and in these directions the course of suppurative diseases of the urine passages may be importantly and favourably acted upon. And this brings me to speak of some of the means at our disposal for rendering the urine sterile so far as the most important factors in suppurative diseases are concerned. I have already referred to the sterilising influence that certain oils and essences, such as sandal wood and copaiba, are capable of exercising in this way. No less marked is the effect that boracic acid taken internally is capable of effecting in controlling bacterial life in the urine. Probably no better proof of this exists than in the results observed in connexion with operations on the urethra, such as the passing of catheters and in internal urethrotomy. The late Dr. Palmer, of Louisville, showed many years ago that by the nse of boracic acid beforehand the development of urethral rigors and fever, under these circumstances, was rendered highly improbable, and this has since been amply proved to be the case. Similarly the use of quinine as a prophylactic against urine fever has been shown to be most efficacious; salol and other like drugs are also well recognised as urine sterilisers. Though boracic acid in doses of from ten to fifteen grains three or four times in the day is probably one of the most reliable sterilisers, it so often disagrees and causes dyspepsia as to interfere greatly with its use for the purpose. Some years ago my attention was called to a preparation called borocitrate of magnesia, which was described by Dr. Koehler, of Kosten, ${ }^{4}$ in Germany, as a solvent for uric acid calculi, and gravel. In the communication referred to it is stated that it is prepared by dissolving boracite, a natural borate of magnesia which is found in Stassfurt, in citric acid. Whether it is actually a solvent for uric acid I am not prepared to say. Whilst testing it for this purpose I found that it frequently had a remarkable effect in sterilising and clearing up purulent urine and in favouring the expulsion of calculi and gravel by its flushing effects on the urine passages. For the latter purpose I have used it for the last ten years in conjunction with other means for preventing urine decomposition with considerable effect. I am in the habit of prescribing it in teaspoonful doses in half a tumbler or so of either warm or cold water two or three times in the day. It is extremely palatable to take and readily dissolves. We have other means for sterilising the urine by the mouth which I will mention, as one method or one drug is not universally applicable. There is a combination of the salicylate of soda with the benzoate of soda which possesses this power in a marked degree. I have frequently found urine which has been rendered opaque by the presence of pus and was swarming with bacteria rendered clear in the course of a few days after the use of this preparation. I usually prescribe fifteen grains of both of these salts to be taken together three times a day in an ounce of chloroform water. The hyposulphite of soda in half-drachm doses has also in some instances effected the same object. The principle of urine sterilisation may, however, be extended to other purulent or bacterial conditions of the urinary apparatus. I refer more particularly to some of those chronic suppurations resulting from the formation of abscesses in connexion with the prostate and prostatic urethra, where the pus was originally discharged, sometimes against gravity, into the latter canal. These are often most difficult to heal by reason of the absence of a free and dependent opening. Yet, on the other hand, the opening of the perineum and the incising of the prostate to secure free drainage of the discharge is a proceeding that cannot always be undertaken without some degree of risk in elderly subjects. The thorough cleansing of these deep parts by the use of antiseptics in the form of auto irrigation, as described in the earlier part of this paper, will be found efficacious in many of these cases of chronic suppuration. In some instances of prostatic suppuration it is almost impossible to flush a sinus connected with this part so long as a catheter of any kind is retained in the bladder. On the other hand, this object may be often readily effected by the spontaneous contraction of a bladder more or less distended with fluid. In the sinuses, for instance, arising out of tuberculous prostatitis this method usually proves very effectual. Again, I have resorted to this way of flushing urinary fistulæ with some antiseptic fluid when the primary cause of them has been removed, as by gradual dilatation or after internal urethrotomy. Tortuous routes through the perineum may, by washing out through the medium of the bladder two or three times if necessary in the twenty-four hours, be thus healed. A few months ago I saw a middleaged man with a tight stricture in the deep urethra which only admitted a No. 4 bougie. In addition there were two chronic perineal fistulæ-one opening in the left buttock and the other by the side of the scrotum, through which almost the whole of the urine was passed at the time of micturition. The patient's condition was an extremely distressing one, as it was impossible for him to pass urine with any degree of comfort except upon a water closet. I divided the stricture from within by Maisonneuve's urethrotome. A catheter was then tied in the bladder for forty-eight hours, and on its removal a No. 12 silk catheter á boule was introduced morning and evening. After washing out by means of this in the ordinary way the bladder was finally filled with about a pint of warm boracic lotion, which the patient was required to void naturally in the standing position on the catheter being withdrawn. The fluid escaped as in the ordinary act of micturition with this patientnamely, partly by the urethra and partly through the false routes. The proportion of the fluid coming through the 
urethra gradually increased, whilst that passing through the two fistulæ grew less daily, and in the course of ten days entirely ceased. This method of procedure I have found from repeated trials to importantly contribute in promoting the sound healing of these sinuses and the comfort and cleanliness of the patient.

I have thus endeavoured to bring under notice, as briefly as possible, some ways of influencing urine in connexion with certain specific and non-specific suppurations of the urinary organs, from the kidneys downwards.

Lower Berkeley-street, W.

\section{ON SUDDEN OR UNEXPECTED DEATH FROM NATURAL CAUSES.}

By J. DIXON MANN, M.D. ST. AND., F.R.C.P. LoND ,

PHYSICLAN TO THE SALFORD ROYAL hOSPTTAL; PROFESSOR OF FORENBIO MEDICINE IN OWENS COLLECTE; KXAMINER IN FORENSIC MEDICINE IN THE UNIVERSITY OE LONION AND IN THE VICTORIA UNIVTRSITX.

SudDen death from natural causes, especially when it occurs under suspicious circumstances, is not infrequently the subject of medico-legal investigation. It is, therefore, necessary that medical practitioners should be familiar with the conditions under which sudden death is usually encountered, since the investigation of cases of this kindoften intrinsically difficult-may, in the absence of some previous knowledge of the subject, prove extremely perplexing.

Cases of sudden death from natural causes may be classified under three heads: (1) deaths which are due to the presence of a disease which is universally recognised as one liable to suddenly terminate fatally-for the most part such cases yield satisfastory post-mortem evidence as to the cause of death; (2) deaths due to the presence of a disease which, when fatal, does not usually end life abruptly, and which, consequently, is not generally regarded as a cause of sudden death-in such cases post-mortem examination frequently yields inferential rather than conclusive evidence as to the cause of death; and (3) deaths which do not result from any ascertainable disease, and as to which consequently no evidence whatever is afforded by post-mortem examination.

1. Roughly speaking, about one-half of the total number of cases of sudden death from natural canses in adults is, more or less, due to heart disease, which has existed for some time, and in which no furthur change is in progress at the time of death-such as valvular disease, angina, fatity heart, and sclerosis of the cardiac muscle from chronic myocarditis. In many cases concurrent lung or kidney disease complicates the statistics, such cases frequently being tabulated as deaths solely due to heart disease. Spontaneous rapture of the heart, mostly in men, may exceptionally occur; the left ventricle, often towards the front, is almost invariably the seat of the rupture. It is to be remembered that in traumatic rupture of the heart the right side, usually the auricle, suffers more frequently than the left in the proportion of about as 70 is to 54. Apoplexy and other cognate brain lesions rank second as natural causes of sudden death. It is to be noted that miliary aneurysms of the vessels of the brain, although most common in persons past middle life, occasionally occur in young people, even in children, and by bursting cause death from apoplexy. Chronic alcoholism, a potent factor among the causes of sudden death, is frequently associated with rapidly terminating heart and brain disease. Asphyxia, a not unfrequent cause, may be due to cedema of the glottis, membranous deposit in the trachea, pressure of a neoplasm on the trachea, spasm of the vocal cords, pulmonary embolism, air embolism rupture of a vessel or of an aneurysm into the air-passages, asthma, whooping-cough, pneumo- and hæmo-thorax, pleuritic effusion, and epilepsy. The rupture of a gastric or of an intestinal ulcer, of an aneurysm, of a varicose vein, of the surroundings of an ectopic gestation, the formation of a peri-uterine hæmatocele, may severally prove quickly fatal. Nephritis (uræmia and apoplexy), diabetes, exophtbalmic goitre, and Addison's disease may also terminate with unexpected rapidity. Hæmorrhage into the pancreas occasionally causes sudden death, apparently from the impression produced on the contiguous nerve centres. It is most common in males over forty years of age, who may up to the occurrence of the hæmorrhage to all appearance be in perfect health. Obesity, the habitual nse of alcohol, and the presence of heart disease appear in many of the cases to be predisposing causes. Koetschau, ${ }^{1}$ however, observed bæmorrhage into the pancreas in a woman-an alcoholic-in her twenty-fourth year. Occasionally it occurs in spare people who are free from obvious disease and who are abstemious as regards alcohol. The sufferer may die within half an hour after the occurrence of the hæmorrhage or he may survive for twenty-four or even thirty-six hours. Draper ${ }^{2}$ records five cases between the ages of twenty-six and fifty-five years, of which three were men and two women. Fitz ${ }^{3}$ tabulated sixteen cases, of which eleven were males between thirty-one and seventy years of age, and five were females between twenty-six and forty-seven years. Sudden death has in several instances followed spontaneous rupture of an enlarged spleen, the result of tropical malarial influences, the individual immediately before rupture being to all intents and purposes quite well. Pellereaux ${ }^{1}$ gives the history of thirteen cases of rupture of the enlarged spleen; in five the rupture was spontaneous, and in the remainder it was due to apparently inadequate causes, such as a simple fall in the street. It is to be borne in mind that when the spleen is thus enlarged a mere pat with the palm of the hand may determine rupture and consequent speedy death, which, in the absence of a knowledge of the experience of others, might readily be assumed to be the result of criminal violence.

It is to be remembered that some of the above-named diseases may exist without giving rise to any symptoms until the fatal moment arrives; this applies with special force to diseases which have a prolonged course, during which, as a rule, symptoms indicative of the disease declare themselves. Sach a disease is gastric ulcer. I have seen more than one case in which, until the fatal rupture occurred, absolutely no symptoms were experienced, not even such as might have been attributed to simple dyspepsia; in one such instance a second ulcer was present in the walls of the stomach in addition to the one that ruptured and caused death, and yet until perforation occurred the patient never felt in the stomach any abnormal sensation whatever. As regards some of these diseases, such as spasm of the vocal cords, whooping-congh, and asthma, the most careful post-mortem examination may fail to determine the cause of death. Epilepsy may or may not leave characteristic appearances, such as recent injury to the tongue, ecchymoses of the conjunctiva, the face, the reck, and occasionally of the trunk, together with froth (possibly blood-stained) on the lips. Lesser ${ }^{5}$ examined seventeen cases of death from epilepsy and found that the tongue had been bitten in eight, and that in six conjunctival or subcutaneous hæmorrhages on the face, breast, or trunk were present.

2. Certain diseases which, when fatal, do not usually end suddenly may exceptionally do so.-Such are abscesses and tumours of the brain, meningitis, diphtheria, tracheitis, enlarged thyroid, pulmonary apoplexy, pneumonia, phthisis, pneumo-thorax, plearisy, ulcerative endocarditis, old adherent pericardium, pericarditis, volvulus, and other causes of acute intestinal obstruction, peritonitis, gall-stone colic, rupture of an echinococcus in the liver or elsewhere, Bright's disease, influenza, gout, and acute rheumatism. When fully developed several of these diseases are usually inhibitive of locsmotion, but they occasionally occur in a latent or an ambulatory form and terminate suddenly without their presence being even suspected; it is no uncommon event for a man suffering from such a disease to walk unaided into the outpatient department of a hospital and even to die suddenly ere treatment can be adopted. Meningitis may be latent and may lead to sudden death; even pus may be present without giving rise to any symptoms until immediately before death. Ollivier ${ }^{6}$ records the case of a man who was taken ill while at work. He went to bed and died in four hours, and at the necropsy the cause of death was found to be purulent meningitis. Death may suddenly attack a person (especially in childhood) who has middle-ear disease, death

1 Centralblatt fủ allgemeine Pathologie, 1893.

2 Boston Medical and Surgical Journal, 1886. Medical News, 1889. 4 Annales d'Hygiène, 1882.

5 Vierteljahrsschrift fur gerichtliche Medicin, 1888. "Archives Générales de Médecine, 1838. 